\section{Structures of the Cylindrical and Vesicular Micelles of an P4VP-longer Asymmetric PS- $b$-P4VP}

\author{
Nauman Ali, Woo-Han Sul, Dong-Yul Lee, \\ Dong-Hun Kim, and Soo-Young Park*
}

Department of Polymer Science, Kyungpook National University, Daegu 702-701, Korea

Received April 24, 2009; Revised May 27, 2009;

Accepted May 27, 2009

\section{Introduction}

The control of the self-assembled micellar morphology of amphiphilic block copolymers in a selective solvent has engrossed substantial interests in the theoretical and applied research fields. ${ }^{1-3}$ The micellar morphology of the block copolymers can be controlled by solvent selectivity and block copolymer composition. ${ }^{4}$ There are three main factors for controlling these morphologies, i.e., the stretching of core blocks, the repulsive interaction among corona chains, and the surface tension of the core/corona interface at the onset of micellization. ${ }^{5,6}$ The block copolymer can selfassemble into spherical, cylindrical, and vesicular micelles in a given solution. ${ }^{4}$ Among those micelles, the vesicle has fascinated scientists because of its diverse applications such as drug delivery diagnostics, ${ }^{7}$ electronics (chip nanopatterning), ${ }^{8}$ and catalysis (enzyme entrapment). ${ }^{9}$ Vesicles (generally kinetically trapped and non-equilibrium $)^{10}$ are usually formed by low molecular materials such as surfactants or lipids.

Wittmann et al. for the first time reported the polymeric vesicles of poly(styrene- $b$-isoprene) (PS- $b$-PI) and poly(styrene- $b$-ethylene oxide) (PS- $b$-PEO) in a mixture of ethyl benzene (a selective solvent for both blocks) and $n$-decane (a PI and PEO selective solvent). ${ }^{11}$ Schrage et al. investigated vesicular morphologies of quaternized poly(1-methyl-4vinyl pyridine- $b$-styrene) and poly(1,2-butadiene- $b$-(cesium methacrylate)) which were self-assembled in THF (nonselective for PS and poly(1,2-butadiene) but selective for charged blocks). ${ }^{12}$ Kesselman et al. also studied the change in the vesicular morphologies of PS- $b$-PI by controlling solvent selectivity in diethyl phthalate (less PS-selective) and dimethyl phthalate (highly PS-selective) mixtures. ${ }^{13}$ Putaux et al. investigated the vesicular morphologies of PS- $b$-PI in $n$-heptane and $n$-decane, both of which are selective solvents for PI. ${ }^{14}$ The PS- $b$-P4VP vesicles have been observed

\footnotetext{
*Corresponding Author. E-mail: psy@knu.ac.kr
}

when the P4VP blocks were complexed with other small molecules. $^{15-19}$ The vesicles of PS- $b$-P4VP in chloroform were observed when perfluorooctanoic acid (PFOA) was added to be complexed with P4VP. ${ }^{18}$ Peng et al. also reported another interesting research on the vesicular morphology of PS- $b$-P4VP in a complex form with low molecular-weight aliphatic acids (stearic acid, decanoate, acetic acid, and formic acid) in chloroform (non-selective for PS- $b$-P4VP and aliphatic acids but selective in a complex form).$^{20}$ But, PS- $b$ P4VP vesicles without complexation were rarely observed. The cylindrical morphology of an asymmetric PS(19600)$b$-P4VP(5100) (PS longer) in P4VP selective solvents was reported. However, the vesicles with asymmetric PS- $b$-P4VPs (P4VP longer) in PS selective solvents have not been reported. The block length of P4VP of the PS- $b$-P4VP is of prime importance because a number of valuable metals or small molecules could be complexed with the P4VP core of micelles (in the PS selective solvents) which is utilized as a nanoreactor.

The screening of the solvent selectivity is necessary to check the possible micellar morphologies of the given block copolymer. Recently the micellar structures and their ordered structures of PS(12K)- $b$-P4VP(11.8K) and PS(19.6K)- $b$-P4VP $(5.1 \mathrm{~K})$ in the toluene/ethanol solvent system were reported. ${ }^{21,22}$ However, we believe that solvent selection for PS- $b$-P4VP is important in this study. We used the toluene/ethanol solvent system where toluene and ethanol are selective solvents for PS and P4VP, respectively. The toluene/ethanol system covers from PS-selective, to neutral, and to P4VPselective solvents although our previous THF/toluene system covers only from neutral to PS-selective solvents. ${ }^{23}$ Thus, we expected that a wider range of the micellar morphologies can be formed in the toluene/ethanol solvent system. The solvent selectivity was narrowly screened by changing the mixing ratio of the two solvents. But the vesicular structure was not found probably due to the less-asymmetric composition of the block copolymer and/or the less selectivity of the used solvent mixtures. The research in this article used a more-asymmetric block copolymer of PS (3.3K)- $b$-P4VP(18.7 K) (P4VP longer) in the toluene/ethanol mixtures to screen the solvent selectivity.

\section{Results and Discussion}

Figure 1(a) shows the SAXS patterns of the $1 \mathrm{wt} \%$ solutions as functions of $q$ and $\phi{ }^{24}$ Micelles were formed in the narrow range of $\phi=88$ to 94 . The solutions were isotropic and insoluble at $\phi=0$ to 86 and $\phi=96$ to 100 , respectively. This narrow range was due to high asymmetry of the composition of the PS(3.3 K)- $b$-P4VP(18.7 K). The SAXS data at $86 \leq \phi \leq 94$ with $\Delta \phi=2$ (Figure 1(b)) were obtained. Significant difference in the SAXS pattern with the small change 


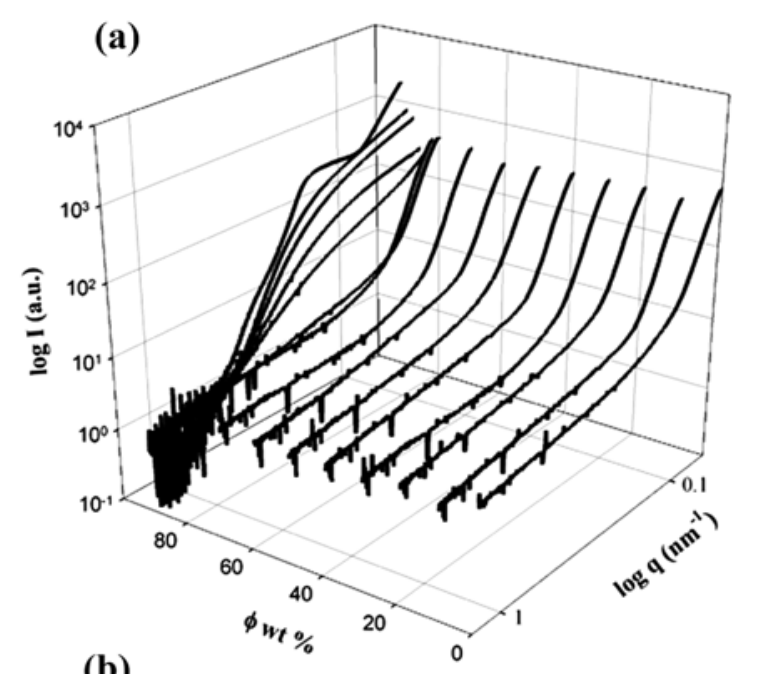

(b)

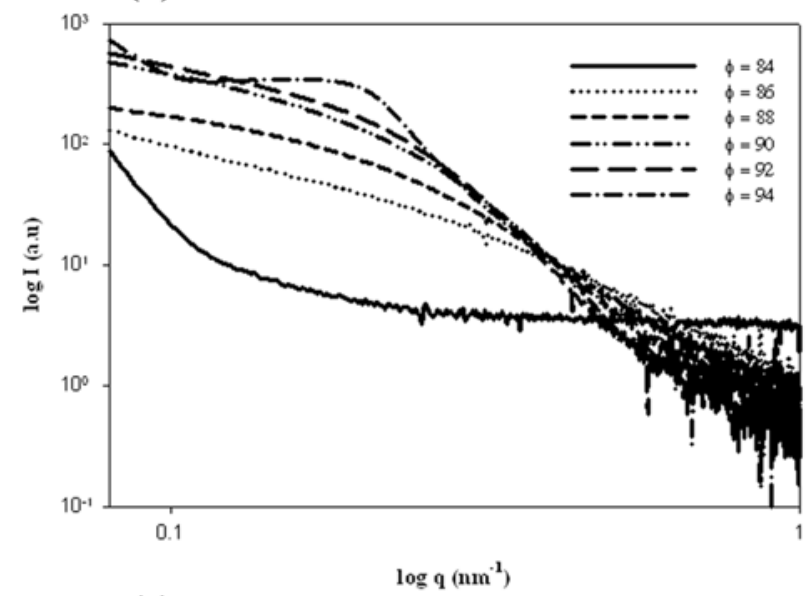

(c)

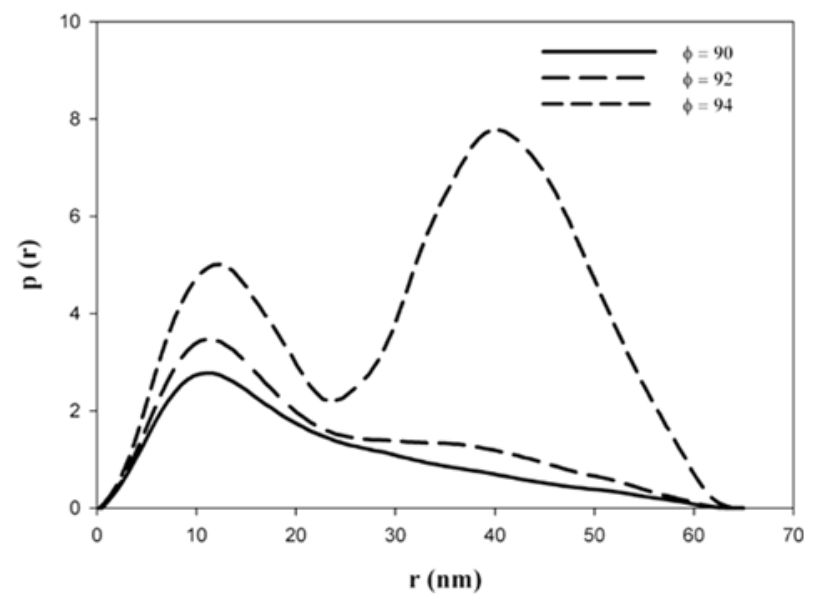

Figure 1. (a) SAXS patterns of the $1 \mathrm{wt} \%$ solutions as functions of $q$ and $\phi$; (b) SAXS patterns at $\phi=86$ to 94 with an interval of 2 in $\phi$, (c) $p(r) \mathrm{s}$ at $\phi=94,92$, and 90 which were calculated from (b).

in $\phi$ indicates that the micellar structure was changed by this small variation in $\phi$ in this range. The SAXS pattern at $\phi=$ 94 showed a steep increase in intensity at low $q\left(0.061 \mathrm{~nm}^{-1}\right.$ $<q<0.098 \mathrm{~nm}^{-1}$ ) with the slope of 3.3 in a log-log scale and had a valley at $q=\sim 0.1 \mathrm{~nm} .{ }^{25}$ The SAXS patterns at $\phi=92$, 90 and 88 were less intense and had slopes of $1.1,1.0$ and 0.73 , respectively, at the same $q$ range with that at $\phi=94 .{ }^{24}$ Figure 1(c) shows the $p(r) \mathrm{s}$ at $\phi=94,92$, and 90 which were calculated from Figure 1(b). The $p(r) \mathrm{s}$ at $\phi=90$ and 92 had a long tail at high $r$, which is typical of a cylinder. The $r$ value at the inflection $(\sim 20 \mathrm{~nm})$ usually represents the diameter of the cylinder. The $p(r)$ at $\phi=94$ shows bimodal distribution with two maxima at $r=\sim 11$ and $42 \mathrm{~nm}$. The first maximum at $r=\sim 11 \mathrm{~nm}$ was the same as those at $\phi=90$ and 92. The $D_{\max }$ (i.e., the maximum dimension of the micelle) is $\sim 63 \mathrm{~nm}$. The shape of $p(r)$ resembles that of a vesicle with a monolayer wall. ${ }^{26-33}$ The occurrence of minima in $p(r)$ signifies the existence of regions of electron density with alternating signs. The alternate signs of the electron density can happen because of the low electron density in the middle of the vesicle.

Figure 2 shows the TEM images of the $0.0156 \mathrm{wt} \%$ PS (3.3 K)- $b$-P4VP(18.7 K) solutions at $\phi=94$ and 86 . The TEM images were prepared by the evaporation of the solutions on the carbon-coated copper grid and stained with $I_{2}$ which selectively stained the P4VP block. These TEM images were taken from the samples evaporated from a very diluted solution so that the separated individual micelles could be observed. Figure 2(a) shows the clear vesicular micelles at $\phi=94$. The size of the vesicle was $\sim 70 \mathrm{~nm}$ in diameter and the wall thickness was $\sim 15 \mathrm{~nm}$. These results were consistent with SAXS data. Figure 2(b) shows the cylindrical micelles with a small population of the vesicular micelles. This change of the micellar structure from cylinder to vesicle reflects the general trend of the morphological transitions of the micelles by increasing the solvent selectivity. The asymmetric PS(3.3)- $b-\mathrm{P} 4 \mathrm{VP}(18.7)$ formed "crewcut" micelles where the corona chains were much shorter than the core chains, which are susceptible to form cylindrical and vesicular morphologies. A sphere-to-cylinder-to-lamella (or vesicle) transition is sometimes observed from the crewcut micelles by increasing solvent selectivity. The size of

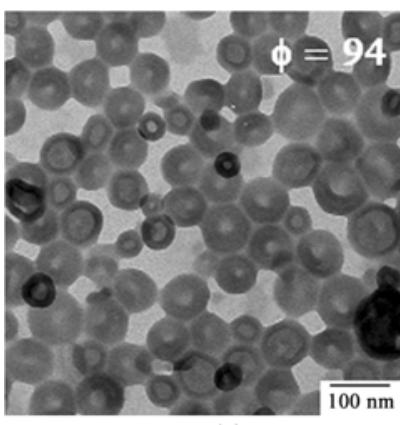

(a)

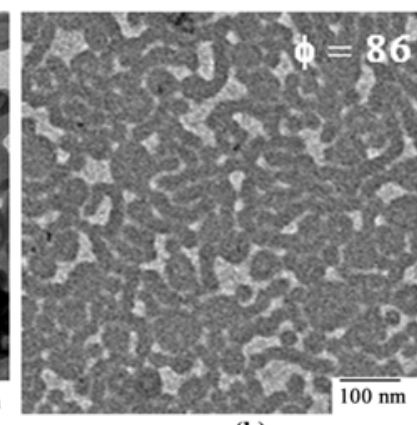

(b)
Figure 2. TEM images of $0.16 \mathrm{wt} \% \mathrm{PS}(3.3 \mathrm{~K})-b-\mathrm{P} 4 \mathrm{VP}(18.7 \mathrm{~K})$ solutions at $\phi=94$ (a) and 86 (b), which were prepared by the evaporation of the solutions on the carbon coated copper grid and stained with $I_{2}$. 
the core is known to increase as solvent selectivity increases in order to reduce the surface tension, so the core chains are stretched in the radial direction compared with their dimensions in the unperturbed state. ${ }^{34}$ The degree of stretching is likely proportional to the radius of the micelle core..$^{35,36}$ This stretching of the core chains decreases as the shape changes from sphere (the ratio of the core radius to the chain end-toend distance in the unperturbed state, $r=1.4)$, to $\operatorname{rod}(r=1.25)$, and to lamella (or vesicle) $(r=1.0)$. Beyond a certain degree of stretching, the morphology changes from sphere to rod, and eventually to lamella (or vesicle), presumably in order to reduce the thermodynamic stretching penalty. However, the repulsive interactions among the corona chains increase as the morphology changes from sphere to rod and to lamella due to increasing crowdedness between the corona chains. For crew-cut micelles, the increase of repulsive interactions in the corona would be smaller than the decrease of stretching energy due to short corona chain length. Thus, more morphological changes can be expected from the crew-cut micelles than the hairy micelles by changing solvent selectivity. ${ }^{23}$ The vesicle of PS- $b$-P4VP was observed in the selective solvent without adding small molecules. This observation might be due to the more systematic screening of the solvent selectivity.

However, it is practically impossible to check all the solvent selectivity. A simple method was found to check the solvent selectivity from a large droplet by one experiment. Figure 3 shows the TEM image of the $1 \mathrm{wt} \% \operatorname{PS}(3.3 \mathrm{~K})-b$ $\mathrm{P} 4 \mathrm{VP}(18.7 \mathrm{~K})$ solution which was evaporated from a large droplet on the carbon-coated copper grid and stained with $I_{2}$. The profound difference in micellar structure was found between the edge and the center of the droplet. Ethanol in

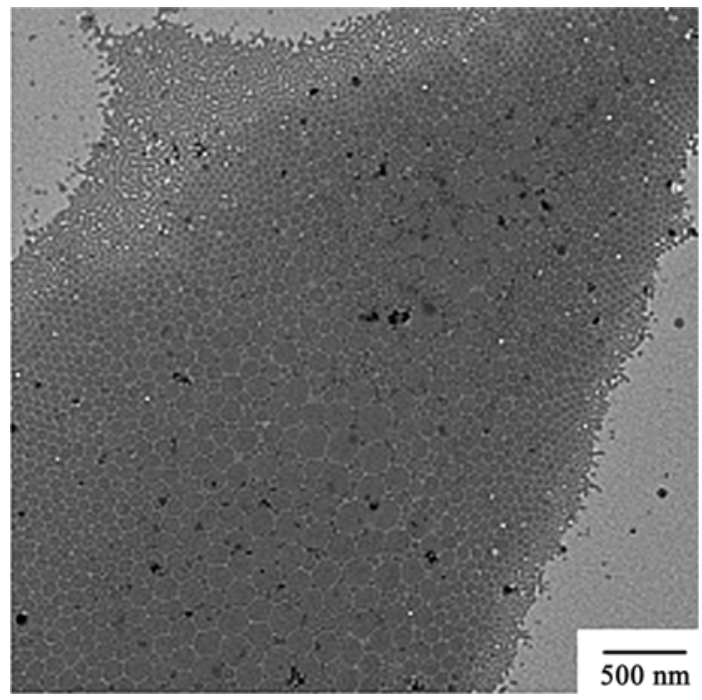

Figure 3. TEM images of a large droplet of the $1 \mathrm{wt} \%$ PS (3.3 K)- $b$-P4VP(18.7 K) solution at $\phi=90$, which were prepared by the evaporation of the solutions on the carbon-coated copper grid and stained with $I_{2}$. the toluene/ethanol mixture would evaporate faster than toluene during drying because of its lower boiling point. The edge in the droplet would dry faster than the center. The evaporation of ethanol in the mixture at the edge would push un-evaporated toluene to the center, so that the toluene content in the center (i.e., the selectivity of the solution) increased from the edge to the center of the droplet during the drying process on the TEM grid. The edge part mostly consisted of cylinders while the center part consisted of only vesicles. The size of the vesicle increased as the position moved to the center of the droplet: the diameter of the vesicular micelle was $\sim 250 \mathrm{~nm}$ in the center as compared to a few tens nm at the edge. A small change in solvent selectivity might influence the curvature of vesicle, and a small curvature change leads to a larger change in size for the vesicular micelle. These results suggest that the library of the micellar structures could be obtained from this simple experiment, which is the same concept of the combinatorial experiment. ${ }^{37}$ From this simple experiment, the possible solvent selectivity was screened and the results were almost the same as those from the separate experiments in Figures 1 and 2 . However, the more systematic study is necessary to set up the procedure for a combinatorial experiment.

In summary, the effect of the solvent selectivity on the micellar structure of an asymmetric PS(3.3)- $b$-P4VP(18.7) diblock copolymer was screened by using a toluene/ethanol mixture. Micelles were formed in the narrow range of $\phi=88$ to 94 ; the solutions were isotropic and insoluble at $\phi=0$ to 86 and $\phi=96$ to 100 , respectively. Cylindrical micelles with a P4VP core were observed at $\phi=90$ and 92. Further increase in the solvent selectivity at $\phi=94$ caused a twodimensional vesicular structure. The vesicular structure was studied with $p(r)$ and was in good agreement with TEM data. It was also found that the structural transition from cylinder to vesicle from the TEM image of a big droplet where the edge part mostly consisted of the cylinder but only vesicles were observed in the center. The vesicle of PS- $b$-P4VP was rarely observed in the PS selective solvent without adding small molecules. This observation might be due to the more systematic screen of the solvent selectivity. This morphological change can be utilized in a given application where specific morphology and dimensions are important.

Acknowledgements. This work was supported by the Korea Research Foundation Grant funded by the Korean Government (MOEHRD, Basic Research Promotion Fund) (KRF-2007-521-D00125) and synchrotron work was supported in part by the Ministry of Science \& Technology (MOST), by POSCO, by the Center for Integrated Molecular System (Korea Science \& Engineering Foundation), and by the KISTEP (Basic Research Grant of Nuclear Energy, MOST). This work was also supported by the second stage of the Brain Korea 21 Project in 2009. 


\section{References}

(1) K. J. T. Noonan, B. H. Gillon, V. Cappello, and D. P. Gates, J. Am. Chem. Soc., 130, 12876 (2008).

(2) Y. J. H. Choi, S. W. Kim, J. H. Kwak, and Y. Tae, Macromol. Res., 16, 609 (2008).

(3) Z. Lei, L. Zhang, and X. Wei, J. Colloid Interf. Sci., 324, 216 (2008).

(4) D. G. Bucknall and H. L. Anderson, Science, 302, 1904 (2003).

(5) L. Zhang and A. Eisenberg, Macromolecules, 29, 8805 (1996).

(6) L. Zhang, K. Yu, and A. Eisenberg, Science, 272, 1777 (1996).

(7) K. Rupert, P. Bidhari, M. Andreas, and T. Marcus, Langmuir, 24, 613 (2008).

(8) B. Schuster, NanoBiotechnology, 1, 153 (2005).

(9) H. Xu, Y. Deng, D. Chen, W. Hong, Y. Lu, and X. Dong, J. Control. Release, 130, 238 (2008).

(10) B. H. Robinson, Self-Assembly, IOS Press, Amsterdam, 2003.

(11) J. C. Wittmann, B. Lotz, F. Candau, and A. J. Kovacs, J. Polym. Sci.: Polym. Phys. Ed., 20, 1341 (1982).

(12) S. Schrage, R. Sigel, and H. Schlaad, Macromolecules, 36, 1417 (2003).

(13) E. Kesselman, Y. Talmon, Y. Bang, S. Abbas, Z. Li, and T. P. Lodge, Macromolecules, 38, 6779 (2005).

(14) J. L Putaux, E. Minatti, C. Lefebvre, R. Borsali, M. Schappacher, and A. Deffieux, Faraday Discuss., 128, 163 (2005).

(15) X. He and F. Schmid, Phys. Rev. Lett., 137802, 137802-1 (2008).

(16) L. Liu, X. Gao, Y. Cong, B. Li, and Y Han, Macromol. Rapid. Commun., 27, 260 (2006)

(17) G. Hou, L. Zhu, D. Chen, and M. Jiang, Macromolecules, 40, 2134 (2007).

(18) H. Peng, D. Chen, and M. Jiang, J. Phys. Chem. B, 107, 12461 (2003).

(19) D. H. Kang, Y. S. Kim, and J. Hak, Macromol. Res., 17, 104 (2009).

(20) H. Peng, D. Chen, and M. Jiang, Langmuir, 19, 10989 (2003).

(21) S. Y. Park, W. H. Sul, and Y. J. Chang, Macromolecules, 40, 3757 (2007).

(22) S. Y. Park and W. H. Sul, Polymer, 49, 3327 (2008).

(23) N. Ali and S. Y. Park, Langmuir, 24, 9279 (2008).

(24) The solution samples were prepared in different $\phi$ (wt $\%$ of toluene in the toluene/ethanol mixture). The sample holder for SAXS has a mica window for X-ray transmission. The solutions were injected into the holder and then the hole was sealed with epoxy in order to prevent evaporation. Experiments were performed at beamline $4 \mathrm{C} 1$ in the Pohang Light
Acceleration Source (PAL, Korea), where a W/B4C doublemultilayer monochromator delivered monochromatic X-rays that had a wavelength of $0.16 \mathrm{~nm}$. A flat Au mirror was used to reject the higher harmonics from the beam. A MarCCD camera (Mar USA, Inc. CCD165) was used to collect the scattered X-rays. The sample-to-detector distance (sdd) was 3 $\mathrm{m}$, which allowed the SAXS data to be obtained in a $\mathrm{q}$ range between 0.06 and $1.11 \mathrm{~nm}^{-1}$ (sdd=3 m). The sdd was calibrated using SEBS (polystyrene-block-poly(ethylene-ran-butylene)-block-polystyrene).

(25) The raw spectra were corrected by conventional procedures for the background of the solvent and sample cell and detector efficiency. Two-dimensional scattering spectra were azimuthally averaged. The measured intensity $I(q)$ can be expressed as the product of form $(F(q))$ and structure $(S(q))$ factors, although $S(q)$ is negligible for the dilute solution. The SAXS data were analyzed with GIFT software, which was developed by Glatter. ${ }^{26-32}$ The Fourier transformation of $I(q)$ yields the pair distance distribution function $(p(r))$ of a particle, which is a histogram of distances inside the particle weighted with the electron density differences. The shape of $p(r)$ allows for the determination of basic geometry (spherical, cylindrical, or vesicular), even for inhomogeneous particles. $D_{\max }$ is the maximum intraparticle distance and $d_{\max }=\pi / q_{\min }$ where $q_{\min }$ is the peak position in the scattering pattern. $D_{\max }$ was determined as the value of $r$ at which $p(r)=0$.

(26) O. Glatter and O. Krathy, Small-angle X-ray scattering, Academic Press, London, 1982.

(27) O. J. Glatter, J. Appl. Crystallogr., 10, 415 (1997).

(28) O. J. Glatter, J. Appl. Crystallogr., 13, 577 (1980).

(29) O. J. Glatter, J. Appl. Crystallogr., 14, 101 (1981).

(30) J. Brunner-Popela and O. J Glatter, J. Appl. Crystallogr., 30, 431 (1997).

(31) A. Bergmann, G. Fritz, and O. J. Glatter, J. Appl. Crystallogr., 33, 1212 (2000).

(32) B. Weyerich, J. Brunner-Popela, and O. J. Glatter, J. Appl. Crystallogr., 32, 197 (1999).

(33) M. R. Brzustowicz and A. T. Brunger, J. Appl. Crystallogr., 38, 126 (2005).

(34) R. Nagarajan and K. Ganesh, J. Chem. Phys., 90, 5843 (1989).

(35) H. Shen and A. Eisenberg, J. Phys. Chem. B, 103, 9473 (1999).

(36) L. Chen, H. Shen, and A. Eisenberg, J. Phys. Chem. B, 103, 9488 (1999).

(37) Y. Zhang, S. Yang, L. Chen, and J. R. G. Evans, Langmuir, 24, 3752 (2008). 